\title{
Safety profile of biologic therapy in Polish paediatric patients with Crohn's disease
}

\author{
Edyta Szymanska1', Maciej Dadalski², Grzegorz Oracz², Jarosław Kierkus² \\ ${ }^{1}$ Department of Paediatrics, Nutrition, and Metabolic Disorders, Children's Memorial Health Institute, Warsaw, Poland \\ ²Department of Gastroenterology, Hepatology, and Feeding Disorders, Children's Memorial Health Institute, Warsaw, Poland
}

Prz Gastroenterol 2015; 10 (3): 164-168 DOI: $10.5114 /$ pg.2015.52298

Key words: biologic therapy, safety, Crohn's disease, children.

Address for correspondence: Edyta Szymanska MD, Department of Paediatrics, Nutrition, and Metabolic Disorders, Children's Memorial Health Institute, Al. Dzieci Polskich 20, 04-730 Warsaw, Poland, phone: +48 513017 570, e-mail: edyta.szymanska@czd.pl

\begin{abstract}
Introduction: In recent years, monoclonal antibodies against tumor necrosis factor $\alpha$ (TNF- $\alpha$ ), infliximab (IFX), and adalimumab (ADA) have gained increasing popularity in Crohn's disease (CD) management. Many clinical trials have shown that biologics are a generally well-tolerated and safe treatment. However, the follow-up time with regards to safety is too short, and data on that issue are still limited.

Aim: To report the cumulative safety profile of biologic therapy with IFX and/or ADA, up to 8 years, in Polish children with moderately to severely active CD.

Material and methods: We performed a retrospective analysis of 110 children, aged $13.0 \pm 9.3$ years, diagnosed with CD, and treated with IFX and/or ADA, within a period of 8 years between 2005 and 2013. Safety data for all treated patients were collected throughout the entire treatment period and were included in the safety analyses.

Results: The cumulative rates of treatment-related adverse events (AE) (TRAEs) in all patients were 67 events -43 (64.17\%) events for IFX and 24 (35.83\%) for ADA, respectively. The majority of TEAEs were mild-to-moderate in intensity. The most frequently reported ones were: anaemia in 17 (20.23\%) IFX patients and 9 (23.08\%) ADA patients, and mild infections in 9 (10.7\%) IFX patients and 5 (12.8\%) ADA patients, respectively. We did not report any serious AE (SAE).

Conclusions: Biologic therapy with infliximab and/or adalimumab is generally well tolerated and safe, and does not cause any SAEs.
\end{abstract}

\section{Introduction}

Crohn's disease (CD) is an inflammatory condition of unknown aetiology, which is classified in the inflammatory bowel disease (IBD) group along with ulcerative colitis (UC) [1, 2]. Since no effective CD treatment protocol has been developed [3], the goals of both pharmacotherapy and surgical management are limited to obtaining the longest possible remission and preventing relapses [4].

In recent years, however, the advent of biological drugs has had a significant impact on the management of IBD. Many clinical trials have demonstrated the efficacy and safety of monoclonal antibodies against anti-tumour necrosis factor (TNF), infliximab (IFX), and adalimumab (ADA) in both induction [5] and maintenance [6] of clinical remission. Moreover, treatment with biologics has also led to endoscopic healing [7, 8], which appears to indicate a longer duration of clinical remission $[9,10]$.

Nonetheless, the use of biologic agents is still limited, and the most frequently cited reasons for this are the high cost and uncertainty about long-term safety. The safety issue is especially important. Although biologics are generally considered to be well-tolerated and safe, the follow-up time with regards to safety is too short and data on that issue are still limited. The safety concerns associated with biologic therapies include the increased risk of infection, autoimmunity, development of lymphoma and demyelinating disease, and the risk of worsening heart failure [11-13]. Reactivation of mycobacterial infections with anti-TNF agents has also been described [14].

The monoclonal antibodies used for anti-TNF therapy frequently induce the formation of antibodies: anti- 
bodies to infliximab (ATI) and antibodies to adalimumab (ATA). The presence of these antibodies was associated with a shorter duration of response to therapy and a higher rate of infusion reactions [15].

The safety of IFX has been evaluated by the TREAT registry, which was established to study the long-term safety of this biologic and other therapies in prospectively followed patients with CD [16]. There have been 6290 patients (3179 IFX recipients and 3111 recipients of other therapies) enrolled in this registry since August 2004. According to available data, the general overall safety of IFX is similar to that of conventional IMM $[9,17]$.

Nonetheless, the data from Central Europe concerning the safety of biologic therapy, especially of ADA, are still limited.

\section{Aim}

Therefore, we would like to report the cumulative safety profile of biologic treatment with IFX and/or ADA in Polish children with moderately to severely active CD.

\section{Material and methods \\ Patients}

We performed a retrospective analysis of children with moderately to severely active CD treated with biologic therapy with IFX and/or ADA at the Department of Gastroenterology, Hepatology, and Feeding Disorders, Children's Memorial Health Institute. The analysis was carried out over an 8-year period - between 2005 and 2013. Table I contains the patients' detailed characteristics.

\section{Safety evaluation}

Adverse events were monitored throughout the entire treatment period. Safety data were reported for all patients, by prior biologic use, as well as by concomitant immunomodulatory agent (IMM) and corticosteroid use at baseline of biologic therapy, and were included in the safety analyses. Adverse events AEs that occurred on or after the first biologic agent dose and up to 70 days after the last dose were considered related to treatment (TEAEs).

\section{Results}

The cumulative rates of TRAEs in all 110 patients were 67 events - $43(64.17 \%)$ events for IFX and 24 (35.83\%) for ADA, respectively. The most common TRAEs were anaemia in 17 (20.23\%) IFX patients and $9(23.08 \%)$ ADA patients, and mild infections in $9(10.7 \%)$ IFX patients and 5 (12.8\%) ADA patients, respectively. Upper respiratory tract infections were the most frequently reported infections for IFX, and gastro-intestinal infections for ADA, 4/9 (44.44\%) vs. 2/5
(40.0\%), respectively. There were no incidents of serious infections. There were no deaths, malignancies, central nervous system demyelinating disorders, optic neuritis, or seizures during the biologic therapy period. Infusion reactions were observed in 3 IFX patients (4.5\%). There were no cases of serum sickness-like reactions or possible delayed hypersensitivity reactions. No AEs leading to the termination of therapy were observed. Table II contains total exposure to IFX and ADA.

We have not observed any connection between higher rates of AEs and prior exposure to IFX: AEs rate/ number of patient (ratio): 17/26 pt (0.65) vs. 7/13 patients (ratio 0.53) for naïve and prior exposed patients, respectively.

\section{Discussion}

Biologics are generally considered well-tolerated and safe. However, the follow-up time with regards to safety is too short and data on that issue are still limited. Besides, there is a tendency towards under-reporting of less severe health problems such as skin eruptions, fatigue, and general malaise.

According to literature, the most common side effects reported for biologic therapy are infections [18], with upper respiratory tract and urinary tract infections as the most frequently reported ones [19]. Since viral infections such as cytomegalovirus and Epstein-Barr virus infections have been predominantly connected with common IMM therapy, biologics are more frequently associated with bacterial opportunistic infections such as tuberculosis, histoplasmosis, invasive aspergillosis, and others [20, 21]. The incidence of opportunistic infections in different groups of patients treated with IFX was reported to vary between $0.3 \%$ and $0.9 \%$ [22], and their occurrence was associated mainly with the use of concomitant treatment with either corticosteroids or azathioprine (AZA)/6-mercaptopurine [23]. However, the combination of a biologic with IMM did not increase the risk significantly. In our analysis infections were the second most common AEs for both IFX and ADA, and they were reported for 9 patients in IFX and 5 cases in ADA. Upper respiratory tract infections were the most frequently observed ones for IFX, and gastro-intestinal infections for ADA. There was only one case of opportunistic infection - oral candidiasis in a patient treated with IFX and concomitant therapy with AZA, whereas none was reported for ADA.

In the IMAgINE 1 study, which evaluated the safety and efficacy of ADA double-blind maintenance dosing regimens following open-label induction for paediatric patients with moderate to severe $C D$, haematologic AEs were mostly anaemia - 22 out of 39 events [24]. The results of our analysis are consistent with those 
Table I. Detailed characteristics of CD patients treated with biologic therapy $(n=110)$

\begin{tabular}{|c|c|}
\hline Parameter & Results \\
\hline \multicolumn{2}{|l|}{ Gender, $n(\%)$ : } \\
\hline Males & $54(50.5)$ \\
\hline Females & $53(49.5)$ \\
\hline Age, mean \pm SD [years] & $13.0 \pm 9.3$ \\
\hline Duration time of disease, mean \pm SD [years] & $8.4 \pm 7.3$ \\
\hline PCDAl, mean \pm SD & $52.5 \pm 27.5$ \\
\hline \multicolumn{2}{|l|}{ Involved region (\%): } \\
\hline Caecum (L1) & 12.1 \\
\hline Left side (L2) & 31.8 \\
\hline Ileocolon (L3) & 56.1 \\
\hline Upper disease (L4) & 14.8 \\
\hline \multicolumn{2}{|l|}{ Behaviour, $n(\%)$ : } \\
\hline B1 & $93(84.5)$ \\
\hline B2 & $2(1.9)$ \\
\hline B3 & $15(13.6)$ \\
\hline SES-CD (ranges) & $18(0-22)$ \\
\hline \multicolumn{2}{|l|}{ Extraintestinal manifestations (18): } \\
\hline Arthralgia/arthritis & $14 / 18(77.8 \%)$ \\
\hline Osteoporosis & $2 / 18(11.1 \%)$ \\
\hline Erythema nodosum & $2 / 18(11.1 \%)$ \\
\hline \multicolumn{2}{|l|}{ Concomitant treatment, $n(\%)$ : } \\
\hline $\mathrm{AZA}$ & $52(38.2)$ \\
\hline MTX & $9(8.2)$ \\
\hline GKS & $59(53.6)$ \\
\hline
\end{tabular}

of Hyams et al., since anaemia was not only the most common haematological AE but also the most frequently reported AE generally. However, most patients presented with anaemia or other haematologic AEs such as leucopaenia also had concomitant treatment with either IMM or corticosteroid. Therefore, it is possible that their occurrence was associated with the use of concomitant medication rather than with the biologic agent. Besides, one third of IBD patients suffer from recurrent iron deficiency anaemia [25], which may explain the fact that this haematological disorder was the most common AE reported in our analysis. It is even more plausible because 4 out of 17 patients (23.5\%) presenting with anaemia during biologic therapy had had it before the treatment was introduced. However, the anaemia caused by the disease (before the biologic agent was given) was milder and did not involve blood transfusions - contrary to that observed after biologic agent administration.
Table II. Rates of adverse events of interest. Cumulative rate for both IFX and ADA = 67 events

\begin{tabular}{|c|c|c|}
\hline Parameter & $\begin{array}{c}\text { Infliximab, } \\
n(\%)\end{array}$ & $\begin{array}{c}\text { Adalimumab, } \\
n(\%)\end{array}$ \\
\hline$A E(\%)$ & $43(64.17)$ & $24(35.83)$ \\
\hline Any $A E$ & 43 & 24 \\
\hline Serious AE & 0 & 0 \\
\hline AE leading to discontinuation & $3^{*}$ & 0 \\
\hline Infections: & $9(10.7)$ & $5(20.8)$ \\
\hline $\begin{array}{l}\text { Upper respiratory tract } \\
\text { infections }\end{array}$ & $4(44.4)$ & $0(0.0)$ \\
\hline $\begin{array}{l}\text { Lower respiratory tract } \\
\text { infections }\end{array}$ & $2(22.2)$ & $2(40.0)$ \\
\hline Gastro-intestinal infections & $1(11.1)$ & $3(60.0)$ \\
\hline Opportunistic infection: & $1(2.3)$ & $0(0.0)$ \\
\hline Oral candidiasis & $1(1.0)$ & $0(0.0)$ \\
\hline Hematologic AE: & $23(53.5)$ & $11(24.9)$ \\
\hline Anaemia: 17 & $17(73.9)$ & $9(81.8)$ \\
\hline Leucopaenia: 5 & $5(21.7)$ & $2(18.2)$ \\
\hline Neutropaenia: 1 & $1(4.4)$ & $0(0.0)$ \\
\hline Injection site reactions: & 8 (18.6) & $1(4.2)$ \\
\hline Anaphylactic shock: 3 & $3(37.5)$ & $0(0.0)$ \\
\hline Other infusion reactions & $3(37.5)$ & $1(100)$ \\
\hline Urticaria & $2(25.0)$ & $0(0.0)$ \\
\hline Other: & $3(6.9)$ & $2(8.3)$ \\
\hline Elevated transaminases & $1(33.3)$ & $1(50.0)$ \\
\hline Lymphadenopathy & $1(33.3)$ & $0(0.0)$ \\
\hline Positive tuberculin test & $1(33.3)$ & $0(0.0)$ \\
\hline Fever & $0(0.0)$ & $1(50.0)$ \\
\hline
\end{tabular}

*Due to anaphylactic shock switching to ADA.

The overall percentage of infusion reactions with IFX was reported to be $6.1 \%$ [26], including a burning sensation, itching, erythema, and pain. The estimated incidence of serious adverse reactions such as shortness of breath, hypotension, or stridor was 1.0\%. Rarely, a genuine allergic reaction occurs, which is characterised by shortness of breath and urticaria [26]. Our analysis has revealed eight (7.27\%) incidences of infusion reactions in 110 patients, which is consistent with the international data. Anaphylactic shock was reported in 3 patients treated with IFX. In these cases, the infusion was stopped and switched to ADA. The other reactions were mild and effectively treated with a single-dose of hydrocortisone, and most patients were re-challenged after the appropriate precautions.

There have been studies and case series that showed an increased risk of lymphomas related to bi- 
ologic therapy, especially when concomitant treatment with thiopurines is administered [27-29]. However, the problem of malignancies concerns mostly internal medicine gastroenterologists, since the process of carcinogenesis needs time. We have not reported any case of cancer or lymphoma in our patients treated with anti-TNF agents, which is consistent with reports by other authors. A systematic review by Dulai et al. performed to quantify the incidence of serious infection, lymphoma, and death among paediatric patients with IBD who received anti-TNF therapy, has demonstrated no greater risk of lymphoma among children with IBD who received anti-TNF therapy comparing to those treated with other IBD therapies or adults treated with anti-TNF agents. Nonetheless, we are aware of losing our patients to follow-up when they become of age and switch to an internal disease gastroenterologist. This is the time at which they might develop treatment related malignancies due to prolonged use of immunosuppres sive or biologic agents.

We did not observe any serious AEs in the course of biologic therapy, which can be considered as a great success. To the best of our knowledge, the best way of preventing serious complications is appropriate qualification of patients to treatment, based on a complete diagnostic investigation including a chest $\mathrm{X}$-ray and tuberculin test, and careful AE monitoring.

\section{Conclusions}

Biologic therapy with IFX and/or ADA is generally well tolerated and safe, and does not cause any sAEs. However, since severe side effects have been reported, careful consideration and monitoring is required.

\section{Conflict of interest}

The authors declare no conflict of interest.

\section{References}

1. Hanauer SB. Inflammatory bowel disease. N Engl J Med 1996; 334: 841-8

2. Kustosz P, Durlik. Molecular pathogenesis of Crohn's disease. Prz Gastroenterol 2013; 8: 21-6.

3. Benchimol El, Fortinsky KJ, Gozdyra P, et al. Epidemiology of pediatric inflammatory bowel disease: a systematic review of international trends. Inflamm Bowel Dis 2011; 17: 423-39.

4. Dignass A, Van Assche G, Lindsay JO, et al. The second European evidence-based consensus on the diagnosis and management of Crohn's disease: current management. J Crohns Colitis 2010; 4: 28-62.

5. Hyams J, Crandall W, Kugathasan S, et al. Induction and maintenance infliximab therapy for the treatment of moderate-to-severe Crohn's disease in children. Gastroenterology 2007; 132: 863-73.
6. Hyams JS, Lerer T, Griffiths A, et al. Long-term outcome of maintenance infliximab therapy in children with Crohn's disease. Inflamm Bowel Dis 2009; 15: 816-22.

7. D'Haens G, Van Deventer S, Van Hogezand R, et al. Endoscopic and histological healing with infliximab anti-tumor necrosis factor antibodies in Crohn's disease: a European multicenter trial. Gastroenterology 1999; 116: 1029-34.

8. Kierkus J, Dadalski M, Szymanska E, et al. The impact of infliximab induction therapy on mucosal healing and clinical remission in Polish pediatric patients with moderate-to-severe Crohn's disease. Eur J Gastroenterol Hepatol 2012; 24: 495-00.

9. Rutgeerts P, Vermeire S, Van Assche G. Mucosal healing in inflammatory bowel disease: impossible ideal or therapeutic target? Gut 2007; 56: 453-5.

10. Łodyga M, Eder P, Bartnik W, et al. Special paper Guidelines for the management of Crohn's disease. Recommendations of the Working Group of the Polish National Consultant in Gastroenterology and the Polish Society of Gastroenterology. Prz Gastroenterol 2012; 7: 317-38.

11. de Silva S, Devlin S, Panaccione R. Optimizing the safety of biologic therapy for IBD. Nat Rev Gastroenterol Hepatol 2010; 7: 93-101.

12. Lewis JD, Bilker WB, Brensinger C, et al. Inflammatory bowel disease is not associated with an increased risk of lymphoma. Gastroenterology 2001; 121: 1080-7.

13. Van Assche G, Van Ranst M, Sciot R, et al. Progressive multifocal leukoencephalopathy after natalizumab therapy for Crohn's disease. N Engl J Med 2005; 353: 362-8.

14. Siegel CA, Hur C, Korzenik J, et al. Risks and benefits of infliximab for the treatment of Crohn's disease. Clin Gastroenterol Hepatol 2006; 4: 1017-24.

15. Baert F, Noman M, Vermeire S, et al. Influence of immunogenicity on the long-term efficacy of infliximab in Crohn's disease. N Engl J Med 2003; 348: 601-8.

16. Lichtenstein GR, Cohen R, Feagan B, et al. Safety of infliximab and other Crohn's disease therapies-updated TREAT registry data with over 10,000 patients-years of follow-up. Gastroenterology 2005; 128: W1034.

17. Colombel JF, Loftus EV, Tremaine WJ, et al. The safety profile of infliximab in patients with Crohn's disease: the Mayo clinic experience in 500 patients. Gastroenterology 2004; 126: 19-31.

18. Blonski W, Lichtenstein GR. Complications of biological therapy for inflammatory bowel disease. Curr Opinion Gastroenterol 2006; 22: 30-43.

19. D'Haens G. Risks and benefits of biologic therapy for inflammatory bowel diseases. Gut 2007; 56: 725-32.

20. Lichtenstein GR, Feagan BG, Cohen RD, et al. Serious infections and mortality in association with therapies for Crohn's disease: TREAT registry. Clin Gastroenterol Hepatol 2006; 4: 621-30.

21. Sandborn WJ, Faubion WA. Biologics in inflammatory bowel disease: how much progress have we made ? Gut 2004; 53: 1366-73.

22. Toruner M, Loftus EV, Colombel JF, et al. Risk factors for opportunistic infections in inflammatory bowel diseases: a case-control study. Gastroenterology 2006; 130: A71.

23. Colombel JF, Sandborn WJ, Reinisch W, et al. Infliximab, azathioprine, or combination therapy for Crohn's disease. N Engl J Med 2010; 362: 1383-95. 
24. Hyams JS, Griffiths A, Markowitz J, et al. Safety and efficacy of adalimumab for moderate to severe Crohn's disease in children. Gastroenterology 2012; 143: 365-74.

25. Gasche C, Lomer MCE, Weiss G. Iron, anaemia, and inflammatory bowel diseases. Gut 2004; 53: 1190-7.

26. Cheifetz A, Smedley M, Martin S, et al. The incidence and management of infusion reactions to infliximab: a large center experience. Am J Gastroenterol 2003; 98: 1315-24.

27. Beigel F, Steinborn A, Schnitzler F, et al. Risk of malignancies in patients with inflammatory bowel disease treated with thiopurines or anti-TNF alpha antibodies. Pharmacoepidemiol Drug Saf 2014; 23: 735-44.

28. Khan N, Abbas AM, Lichtenstein GR, et al. Risk of lymphoma in patients with ulcerative colitis treated with thiopurines: a nationwide retrospective cohort study. Gastroenterology 2013; 145: 1007-15.

29. Dulai PS, Thompson KD, Blunt HB, et al. Risks of serious infection or lymphoma with anti-tumor necrosis factor therapy for pediatric inflammatory bowel disease: a systematic review. Clin Gastroenterol Hepatol 2014; 12: 1443-51.

Received: 20.10 .2014

Accepted: 18.11 .2014 